\title{
Can Researchers' Personal Characteristics Shape Their Statistical Inferences?
}

\author{
Elizabeth W. Dunn ${ }^{1 *}$, Lihan Chen ${ }^{1}$, Jason D. E. Proulx ${ }^{2}$, Joyce Ehrlinger ${ }^{3}$, Victoria Savalei ${ }^{1}$ \\ ${ }^{1}$ University of British Columbia, \\ Department of Psychology \\ Vancouver, BC, Canada \\ ${ }^{2}$ Simon Fraser University, \\ Department of Psychology \\ Burnaby, BC, Canada \\ ${ }^{3}$ Robinhood \\ Menlo Park, CA, USA
}

Word Count: 9,242

Corresponding Author:

Elizabeth Dunn, Department of Psychology, University of British Columbia, 2136 West Mall, Vancouver, B.C., V6T 1Z4. Email: profdunn@gmail.com

(C) 2020 by the Society for Personality and Social Psychology, Inc. This paper accepted for publication at the Personality and Social Psychology Bulletin (PSPB) is not the copy of record and may not exactly replicate the authoritative document published in the SPSP journal. Please do not copy or cite without the authors' permission. The final article is available, upon publication, at https://doi.org/10.1177/0146167220950522.

\section{Acknowledgement:}

We would like to acknowledge the work of Winnie Tse for her efforts in data collection on Study 2 .

\section{Declaration of Conflict of Interests:}

The author(s) declare no other conflicts of interest with respect to the authorship or the publication of this article.

\section{Funding:}

The author(s) disclosed receipt of the following financial support for the research, authorship, and/or publication of this article: The research was supported by the Social Sciences and Humanities Research Council of Canada (SSHRC) Insight Development Grant and a SSHRC Explore Grant to Victoria Savalei. The authors declare no other conflicts of interest with respect to the authorship or the publication of this article. 
PERSONAL CHARACTERISTICS AND STATISTICAL INFERENCES

\begin{abstract}
Researchers' subjective judgements may affect the statistical results they obtain. This possibility is particularly stark in Bayesian hypothesis testing: To use this increasingly popular approach, researchers specify the effect size they are expecting (the "prior mean"), which is then incorporated into the final statistical results. Because the prior mean represents an expression of confidence that one is studying a large effect, we reasoned that scientists who are more confident in their research skills may be inclined to select larger prior means. Across two pre-registered studies with over 900 active researchers in psychology, we showed that more self-confident researchers selected larger prior means. We also found suggestive but somewhat inconsistent evidence that men may choose larger prior means than women, due in part to gender differences in researcher self-confidence. Our findings provide the first evidence that researchers' personal characteristics might shape the statistical results they obtain with Bayesian hypothesis testing. Keywords: Confidence; Gender; Bayesian Inference; Hypothesis Testing
\end{abstract} Received September 27, 2019; revision accepted for publication July 26, 2020 
PERSONAL CHARACTERISTICS AND STATISTICAL INFERENCES

\section{Can Researchers' Personal Characteristics Shape Their Statistical Inferences?}

Scientists typically strive to conduct studies that provide objective reflections of reality (Slaney, 2001). In the wake of the replication crisis, however, we now recognize that scientists who are motivated to obtain a particular finding may employ "researcher degrees of freedom" to bring their statistical results in line with their expectations (Simmons, Nelson, \& Simonsohn, 2011). Specifically, such "p-hacking” can make non-existent effects appear significant and inflate the size of effects reported in scientific articles (Simonsohn, Nelson, \& Simmons, 2014a, 2014b). If we assume that p-hacking was widespread in our field, then it follows that many published effect sizes are over-estimates (e.g., Open Science Collaboration, 2015). As a result, researchers themselves may have been left with inflated expectations about the effect sizes they are likely to obtain. These expectations, in turn, can potentially shape the outcomes of their research. On the one hand, if researchers expect overly large effect sizes, they may underpower their studies, leading them to obtain non-significant results, even if their hypotheses are correct. On the other hand, if researchers predict rather diminutive effect sizes, their proposed work may be less likely to get funded by grant panels (to the extent that smaller effects are perceived as less important or exciting). ${ }^{1}$ While these consequences may be difficult to document, researchers' expectations are both explicit and transparent in one increasingly popular statistical approach: Bayesian hypothesis testing. A central feature of Bayesian hypothesis testing is that expected effect sizes are incorporated into the analyses and affect the resulting statistical conclusions drawn from the study. In the present research, we focused on Bayesian hypothesis testing and examined whether researchers' personal characteristics are linked to the effect sizes they predict.

\footnotetext{
${ }^{1}$ For a candid discussion of how small effects are perceived in our field, see Inbar \& Inzlicht (2020; episode 40; 50:45-1:13:57). They argue that smaller effects may be perceived as less desirable to study (due to the large number of participants required to study them) and as less worthwhile to share with the media, policymakers, and the general public.
} 
PERSONAL CHARACTERISTICS AND STATISTICAL INFERENCES

Although researchers should rely on theory and on the results of relevant past research in predicting effect sizes, it is not always straightforward to determine which past effect sizes to incorporate or how to combine them. Indeed, a registered replication report (RRR) showed that studies testing the same conceptual hypothesis can produce vastly different effect sizes depending on the specific paradigm used (Aknin, Dunn, Proulx, Lok, \& Norton, 2020). Therefore, in making predictions about effect sizes, scientists may rely in part on their own subjective confidence in their research. Because no measure of researcher self-confidence exists, we created a scale asking scientists to rate their agreement with items such as, "I make good choices about which research ideas to pursue" and "When I test a new hypothesis of mine, I feel confident that it will turn out to be right." Researcher self-confidence levels are almost certainly influenced by factors that are directly relevant to predicting effect sizes (including their past success in obtaining large effect sizes), but it is also possible that confidence is shaped by factors that should be irrelevant, including scientists' gender or academic status.

A wealth of research suggests that confidence is related to gender, power, and level of expertise in the relevant domain (see Dunning, 2005 for review). Women are generally more risk-averse than men (Byrnes, Miller, \& Schafer, 1999; Charness \& Gneezy, 2012). Women also routinely express less confidence in their abilities and the products of their work than their male peers (Beyer \& Bowden, 1997; Huang, 2013; Instone, Major, \& Bunker, 1983; Stankov \& Lee, 2014), particularly in the domains of math and science (Ehrlinger et al, 2017; Ellis, Fosdick, \& Rasmussen, 2016; Else-Quest, Hyde, \& Linn, 2010; Micari, Pazor, \& Hartman, 2007). Further, Ehrlinger and Dunning (2003) showed that women expressed less confidence in their scientific abilities than men and, in turn, were less confident in the quality of their answers on a science exam than their male peers (despite performing equally well on the exam). Examining titles and 
PERSONAL CHARACTERISTICS AND STATISTICAL INFERENCES

abstracts in PubMed, Lerchenmueller and colleagues (2019) found that female scientists were

less likely to frame their research in a positive light by using words such as "novel," "excellent," and "robust" compared to their male counterparts. To the extent that women are less confident (i.e., humbler) than men, they may be more cautious about making bold predictions. If this theorizing is correct, then female researchers may predict lower effect sizes than men. Similarly, people with more power tend to be more confident (Min \& Kim, 2013; See, Morrison, Rothman, \& Soll, 2011). As researchers climb the academic ladder, progressing from being graduate students to professors with tenure, they acquire higher status and greater power. As a result, people with higher academic ranks (e.g., professors vs. graduate students) may expect to obtain larger effect sizes, all else being equal.

\section{Bayesian Hypothesis Testing}

In Bayesian hypothesis testing, researchers must specify the effect size they are expecting, which is then incorporated into the final statistical results. Specifically, the researcher specifies a prior distribution, which is a mathematical representation of the researcher's degree of belief in the likelihood of various population effect sizes. This prior distribution serves as the research hypothesis. Once the data are collected, the researcher calculates a statistic called the Bayes Factor. The larger the Bayes Factor, the stronger the evidence for the research hypothesis (as captured by the prior distribution) compared to the null hypothesis of no effect. ${ }^{2}$

The most familiar prior distribution is a normal curve centered on the effect size the researcher considers to be most likely (see Figure 1). For example, a researcher who believes they are studying a large effect (say, Cohen's $d=.70$ ) would express their confidence in the

\footnotetext{
${ }^{2}$ Our explanation of how Bayes Factors work is brief and necessarily oversimplified. For example, other null hypotheses are possible, and priors under the alternative hypothesis can take different shapes. See Rouder et al. (2009) and Dienes $(2011,2014)$ for more thorough treatments. In addition, for a more detailed introduction to Bayes Factors, please see our tutorial on the OSF at https://osf.io/ry49c/?view only=70d409e8f7bb43e2b67abb2c7937dc83.
} 
PERSONAL CHARACTERISTICS AND STATISTICAL INFERENCES

substantial magnitude of their effect by setting the prior mean to .70 . Thus, setting the prior mean is equivalent to guessing an effect size for power analysis, and this step is relatively intuitive. A second step is also required, however: The researcher must select the prior standard deviation, reflecting the degree of uncertainty around the prior mean. For example, if the researcher believes that there is a $95 \%$ chance that the true population effect size falls between .50 and .90 , they would set their prior standard deviation to .10 (see Figure 1). Choosing a large prior mean is an expression of confidence that one is studying a large effect, while choosing a narrow prior standard deviation is an expression of confidence that the most likely effect size (whether big or small) was guessed correctly. In the present research, we asked researchers to select both prior means and prior standard deviations, but to contain Type I error, we only preregistered hypotheses about prior means, which we felt would be more intuitive for researchers previously unfamiliar with Bayesian statistics to understand and apply.

How does the choice of the prior distribution affect the conclusions that researchers draw from their data? First, researchers will obtain a larger Bayes Factor if their prior distribution matches the observed data; in other words, accuracy matters. Second, the size of the prior mean matters: on average, researchers will obtain bigger Bayes Factors if they choose a prior distribution with a larger prior mean (holding accuracy constant). Finally, a smaller prior standard deviation will result in larger Bayes Factors given that the prior mean matches the observed data. ${ }^{3}$ In sum, an accurate prior distribution with a high mean and a narrow standard deviation will yield the largest Bayes Factor.

\section{The Present Research}

\footnotetext{
3 The combined influence of these three factors (accuracy, prior mean, and prior standard deviation) is more complex. For example, if the prior mean is not accurately specified, the impact of a smaller prior standard deviation may be to increase or to decrease the Bayes Factor, depending on the degree of the mismatch between the prior mean and the mean in the observed data.
} 
PERSONAL CHARACTERISTICS AND STATISTICAL INFERENCES

In the present studies, we examined whether researchers' personal characteristics are related to the prior means they select. We expected that more self-confident (i.e., less humble) researchers would set higher prior means. Given that gender and academic rank may be related to researcher self-confidence, we hypothesized that men (vs. women) and academics with higher (vs. lower) ranks would select higher means for their priors. One challenge of testing these hypotheses is that most psychologists have not been trained in the use of Bayesian statistics. Thus, we began by creating an online tutorial to provide active researchers with an introduction to Bayesian hypothesis testing. We conducted a pilot study that included a subset of our measures, enabling us to refine our tutorial and questionnaire; the materials and data are available on OSF. ${ }^{4}$ We then conducted Study 1, recruiting over 450 graduate students and faculty in psychology across North America. We conducted a partial replication in Study 2. Study materials, data, and analysis of both studies are available on the OSF project page. ${ }^{5}$

\section{Study 1}

\section{Method}

Participants. As pre-registered on the $\mathrm{OSF},{ }^{6}$ we aimed to recruit graduate students and faculty members actively conducting research in psychology and other related fields (e.g., marketing) in universities located across the United States and Canada. We used email and relevant listservs to recruit participants. We wanted to collect as large a sample as possible, within the constraints imposed by our funding and by the limited size of the population of interest. Thus, we pre-registered that we would obtain usable data from at least 200 graduate

\footnotetext{
4 Study materials and data for the preliminary study are available at https://osf.io/fasdb/?view_only=f512743c0e95463b960b294855b40464

${ }^{5}$ Study materials, data, and analysis available at https://osf.io/uayxp/?view_only=76e10f26715a446db5bdcfcef92d612f

${ }^{6}$ Pre-registration available at https://osf.io/q4sud/?view only=a63a4d7a89a54ed392e553996aaa4e03.
} 
PERSONAL CHARACTERISTICS AND STATISTICAL INFERENCES

students and 200 faculty members. We were able to attract enough participants to exceed these targets, thereby ensuring that we would have sufficient usable data. We did not conduct any of our analyses until the study was closed.

The final sample consisted of 485 eligible participants (54.8\% graduate students, $26.6 \%$ non-tenured faculty, $18.6 \%$ tenured faculty; $58.6 \%$ female, $41.4 \%$ male; see Table 1 for summary of sample demographics), who completed the study online in exchange for $\$ 25$ Amazon gift cards. As pre-registered, we excluded 42 participants who scored $60 \%$ or lower on the comprehension quiz. Additionally, we excluded 18 participants who had completed the earlier version of the study; this exclusion criterion was not in the pre-registration because we failed to anticipate that some individuals would participate multiple times. Three participants who did not identify as either female or male were not included because our analysis plan entailed treating gender as a binary variable (given our limited population of interest, we did not expect to obtain a sufficient number of participants to treat gender in a more nuanced manner). Finally, we removed the scenario responses of three participants who selected prior means greater than Cohen's $d=2$, as pre-registered.

Researcher confidence scale. To test whether the effects of gender and/or status on the prior distribution were mediated by researcher self-confidence, we created a Researcher Confidence (RC) scale. We created an initial pool of 19 items with high content and face validity for assessing confidence regarding the strength of one's research ideas, skills, and findings. Pilot testing was conducted with 72 researchers (51 students, 14 faculty, 4 postdocs, and 3 who selected "other"; $52.8 \%$ females), who completed the scale at a social and personality psychology conference (SPSP). Based on the comments from the pilot participants and item-total correlations, we re-worded two items and dropped three items from the scale. The final 16-item 
PERSONAL CHARACTERISTICS AND STATISTICAL INFERENCES

RC scale is provided in the Supplementary Material (Table S3). Respondents were asked to indicate how often they experienced each thought or behavior on a 5-point Likert scale from 0 ("Almost never") to 4 ("Almost always"). Because the newly developed RC scale had gone through limited psychometric validation, we pre-registered a plan to also use a trimmed version of the scale, as well as a single key item, as alternative mediator variables in our analyses. We created the trimmed version by fitting a one-factor model and removing items with loadings below .3. The single key item that we reasoned would most directly tap our construct of interest was "My research projects will yield effects that are at least medium in size."

Procedure. Participants completed the study online. Upon providing consent, participants first completed a brief questionnaire to confirm their eligibility. Eligible participants then completed a demographic survey (including questions about their gender ${ }^{7}$ and age) and then indicated the area of psychology they most identified with, their experience with statistics, their familiarity with Bayesian statistics, and the highest degree they had obtained in psychology (see Table 1). Next, participants went through a very non-technical 30-minute (approximately) tutorial on Bayes factors, developed by the research team. Two experts in Bayesian statistics reviewed the tutorial for accuracy prior to the commencement of the study. The tutorial covered the basic concepts of Bayesian inference and gave information and examples on how to set bellshaped priors on effect sizes for the purpose of testing research hypotheses via Bayes Factors. Participants were free to move through the tutorial at their own pace and could review or skip through any concepts. Upon finishing the tutorial, participants were asked to complete a 10-item comprehension quiz to test their understanding of the key concepts covered in the tutorial;

\footnotetext{
7 Participants were asked, "What is your gender?" with response options "Female," "Male," or "Other (please specify)." Participants who chose "Other" were able to write in a free-text response, consistent with current recommendations to enable participants to describe their own gender identity (Lindqvist, Sendén, \& Renström, 2020; Reisner et al., 2015).
} 
PERSONAL CHARACTERISTICS AND STATISTICAL INFERENCES

participants were unable to return to the tutorial upon starting the quiz. Participants who passed the quiz (with over $60 \%$ correct answers) were then asked to select priors for four scenarios that involved hypothetical research studies and for two scenarios involving their own research.

Finally, participants were asked to complete the 16-item Researcher Confidence (RC) scale.

Hypothetical research scenarios. Participants were asked to imagine that they were the lead researcher on an upcoming study and that they believed the hypothesis being tested to be true. In each scenario, some information about effect sizes was given (e.g., average effect sizes in this hypothetical research area, effects sizes from an earlier study). Participants then used an interactive graph to select the mean and/or standard deviation for the bell-shaped prior representing their expectations for each scenario, in standardized effect size units (Cohen's $d$ ). In one scenario, the standard deviation of the prior was provided, and participants were asked to decide on a mean; in another scenario, the mean of the prior was provided, and participants had to select the standard deviation. In the remaining two scenarios, participants were asked to choose both the mean and the standard deviation. Our pre-registered hypotheses concern the means and not the standard deviations (which were included for exploratory purposes); as such, only three hypothetical research scenarios are available to test our primary hypotheses.

Real research scenarios. Next, participants were asked to construct priors relating to their own research. In the first real research scenario, participants were asked to think of a published or unpublished research study they had previously conducted with just two conditions (either a between- or within-subjects design). Participants were told that it should be a study they really cared about — one which provided at least some support for their hypothesis. Participants were then asked to describe the conceptual independent variable (IV) and the dependent variable (DV) for this study, as well as to indicate what area of psychology the study fell into. Finally, 
PERSONAL CHARACTERISTICS AND STATISTICAL INFERENCES

they were asked to imagine conducting a conceptual replication of the earlier study and to set the mean and standard deviation for the prior, in standardized effect size units. In the second real research scenario, participants were asked to think of a research study they would like to conduct, which would test a specific hypothesis that they believe in. Participants were told that it could be a study they were about to conduct, a study proposed in a recent grant application, or a study that they had always dreamed of conducting. Again, participants were told that it could have a within- or between-subjects design and that it should focus on comparing two conditions. Participants then reported the IV, DV, and area of study, and set the mean and standard deviation of their prior.

\section{Results}

Researcher confidence scale analysis. Of the 485 participants, 481 completed the Researcher Confidence scale. Of these, 34 participants had some missing data on the scale. To investigate whether any items had loadings smaller than .3 on the general factor, a 1-factor model was fit to the data using the full-information maximum likelihood (FIML) estimator in the R package lavaan (Rosseel, 2012; version 0.6-3). While the 1-factor model had poor fit (CFI = .767, RMSEA $=.089$, SRMR $=.066$ ), only two items had standardized loadings smaller than .3 . These loadings were .172 and .164 for items 10 and 11, respectively (see Table S3 for wording). Thus, as pre-registered, we computed a "trimmed" scale composite consisting of the 14 best performing items (Cronbach's $\alpha=.83 ; \mathrm{CFI}=.775$, RMSEA $=.101$, SRMR $=.069$ ), as well as the full 16-item RC scale composite $(\alpha=.82)$. When computing composite scores, missing data were dealt with by computing the item score from all available items for a given participant.

Pre-registered analyses. As pre-registered, we examined the prior means specified by the participants for each of the five scenarios that asked the participants to specify prior means: 
PERSONAL CHARACTERISTICS AND STATISTICAL INFERENCES

three hypothetical scenarios and two real-research scenarios. Following our pre-registered analysis plan, we first examined each scenario separately. We also pre-registered that we would create a composite across all five scenarios if there was at least moderate reliability $(\alpha>.5)$, but the actual reliability $(\alpha=.45)$ failed to meet this threshold because the prior means of the three hypothetical scenarios were not sufficiently correlated with each other $(r s=.14, .18, .34)$ and largely uncorrelated with the prior means for the real research scenarios $(r \mathrm{~s}=.02$ to .16 , see Table S4 for the correlation matrix). ${ }^{8}$ We also pre-registered that we would average across the two real-research scenarios if participants' chosen prior means for these scenarios were at least moderately correlated $(r>.30)$, and the actual correlation $(r=.37)$ met this criterion, so we created a composite for the two real-research scenarios. ${ }^{9}$ All regression and mediation analyses were conducted as path analyses in the R package lavaan $0.6-3$, and remaining missing data was handled using FIML with robust standard errors (i.e., MLR estimation in lavaan).

Individual scenarios. We entered gender (female $=0$, male $=1)$ and academic status $(\operatorname{grad}$ student $=-1$, untenured faculty $=0$, tenured faculty $=1)$ into regressions predicting prior means for each of the five scenarios individually. As shown in Table 2, there was no significant effect of gender or academic status on any individual scenario. However, the strongest effects appear to be those of gender on the prior means set for the real-research scenarios. Although there were no significant effects of gender or status for the hypothetical scenarios, we repeated

\footnotetext{
${ }^{8}$ These lower correlations may have resulted from the lower variability in the participant responses to each hypothetical scenario, as most participants anchored their responses to the numbers provided in the scenario. The standard deviations of the prior means in the three hypothetical scenarios were, respectively, .086, .085, and .074, compared to the standard deviations of .222 and .183 for the two real research scenarios.

${ }^{9}$ For each hypothetical scenario, there was only one missing data point. However, 54 participants did not provide a response to one of the real-research scenarios, and another 18 left both scenarios blank. We computed the correlation and created the composite based on the remaining 412 participants.
} 
PERSONAL CHARACTERISTICS AND STATISTICAL INFERENCES

the mediation models for these scenarios as pre-registered. Doing so yielded largely nonsignificant effects (see Table S8).

Real-research composite. As pre-registered, we entered gender $($ female $=0$, male $=1)$ and academic status ( grad student $=-1$, untenured faculty $=0$, tenured faculty $=1$ ) into regressions predicting the average prior mean for the two real-research scenarios. There was no significant effect of academic status $\left(\beta=-.001, p=0.984, b=-.00, \mathrm{CI}_{95}=[-.02, .02]\right)$, but there was a significant effect of gender $\left(\beta=.10, p=.038, b=.04, \mathrm{CI}_{95}=[.00, .07]\right)$. Consistent with our hypothesis, men selected slightly larger prior means $(M=0.44, S D=0.18)$ than did women $(M=0.40, S D=0.15$; illustration in Figure 2). We did not have any hypotheses regarding possible interactions between gender and status and thus we did not include an interaction term, consistent with our pre-registered analysis plan.

Mediator: Self-confidence. Next, we examined whether the effects of gender or status on prior means were mediated by researcher self-confidence. As gender and academic status were mostly independent $\left(\chi^{2}(2)=3.78, p=.151\right)$, we investigated them separately. Following our preregistered analysis plan, we conducted these analyses with (1) the full scale, (2), the full scale removing items with factor loadings below .3, and (3) a single-item that most directly taps our construct of interest: "My research projects will yield effects that are at least medium in size." For shorthand, we refer to these versions as the full scale, trimmed scale, and single-item scale, respectively.

Gender and self-confidence. Using the lavaan package (0.6-3) in $R$, we performed mediation analyses to test whether the relationship between gender and prior means was mediated by researchers' self-confidence (see Figure 3 and the top section of Table 3). Consistent with our regression analyses, we found a weak but significant total effect of gender on 
PERSONAL CHARACTERISTICS AND STATISTICAL INFERENCES

prior means (total effect in Table 3 and Figure 3 ), $\beta=.11, p=.029, b=.04, \mathrm{CI}_{95}=[.00, .07]$.

Examining the trimmed self-confidence scale, we found a significant effect of gender on selfconfidence (path a in Table 3 and Figure 3), whereby men reported higher self-confidence than women, $\beta=.12, p=.006, b=.11, \mathrm{CI}_{95}=[.03, .19]$. Self-confidence, in turn, was strongly related to selecting larger prior means (path $\mathrm{b}$ ), $\beta=.17, p<.001, b=.06, \mathrm{CI}_{95}=[.03, .10]$. Thus, there was a significant indirect effect of gender on prior means via confidence ( $p$ ath $\mathrm{a}^{*} \mathrm{~b}$ ), $\beta=.02, p=$ $.031, b=.01, \mathrm{CI}_{95}=[.00, .01]$. After accounting for confidence, the direct path (c) from gender to prior means remained positive but did not reach statistical significance, $\beta=.09, p=.069, b=$ $.03, \mathrm{CI}_{95}=[-.00, .06]$. This pattern of results is consistent with our hypothesis that men select larger prior means than women at least partially due to greater self-confidence. When we replaced the trimmed scale with the full scale, the results were substantively the same (see Table S7). Using the single-item scale, the results were similar but weaker, with only the effect of selfconfidence on prior means reaching significance (see Table S7); this pattern is as expected given that the reliability of a single item is much lower than the reliability of a longer scale.

Academic status and self-confidence. The overall relationship between academic status and prior means was not significant (the total effect on Table 4 and Figure 4 ), $\beta=.01, p=.859, b$ $=.00, \mathrm{CI}_{95}=[-.02, .02]$. We next examined whether there was an indirect effect of status on prior means through self-confidence. Using the trimmed self-confidence scale, we found that there was a strong effect of status, whereby individuals with higher academic status reported greater self-confidence, $\beta=.30, p<.001, b=.18, \mathrm{CI}_{95}=[.12, .23]$. Self-confidence was related to selecting larger prior means, $\beta=.20, p<.001, b=.07, \mathrm{CI}_{95}=[.03, .11]$. Thus, there was a significant indirect effect of status on prior means via self-confidence, $\beta=.06, p=.001, b=.01$, $\mathrm{CI}_{95}=[.00, .02]$. Given this substantial indirect effect through the hypothesized pathway, one 
PERSONAL CHARACTERISTICS AND STATISTICAL INFERENCES

might expect a significant total effect of status on self-confidence; this overall effect may have failed to emerge in part because there was a small, unreliable, negative direct effect of status on prior means, $\beta=-.05, p=.329, b=-.01, \mathrm{CI}_{95}=[-.03, .01]$, after taking the indirect effect of confidence into account. Taken together, this suggests that higher academic status is linked to greater self-confidence, which in turn promotes the selection of larger prior means-but that confidence is not the only variable that matters in shaping the relationship between academic status and the selection of prior means. We observed substantively identical effects using the full scale, but there was no effect of status on the single-item scale (see Table S8).

Exploratory Analyses. In addition to our pre-registered analyses, we conducted a series of exploratory analyses. Here, we briefly describe the results of selected exploratory analyses that are likely to be of interest. See Supplementary Materials for all performed analyses.

Hypothetical Scenarios. Although there were no effects of gender or status on prior means for the hypothetical scenarios, we repeated the mediation analyses for the real-research scenarios on an exploratory basis for completeness. These results are presented in Table S5 and Table S6.

Subgroup analysis. To examine the effect of gender within each academic group, we repeated the gender mediation analysis separately for graduate students, untenured faculty, and tenured faculty. The total effect of gender on prior means was positive for all three groups, but appeared stronger for graduate students $\left(\beta=.14, p=.051, b=.05, \mathrm{CI}_{95}=[-.00, .10]\right)$ and tenured faculty $\left(\beta=.17, p=.145, b=.06, \mathrm{CI}_{95}=[-.02, .14]\right)$ than untenured faculty $(\beta=.01, p=.890, b$ $\left.=.00, \mathrm{CI}_{95}=[-.05, .05]\right)$.

To examine whether our effects would hold within a more homogenous sample of psychologists, we also repeated our primary analyses looking only within our largest sub-group: 
PERSONAL CHARACTERISTICS AND STATISTICAL INFERENCES

social/personality psychologists $(N=203)$. Consistent with our findings for the whole sample, gender significantly predicted prior means $\left(\beta=.21, p=.004, b=.05, \mathrm{CI}_{95}=[.02, .09]\right)$ and status $\operatorname{did} \operatorname{not}\left(\beta=.09, p=.312, b=.01, \mathrm{CI}_{95}=[-.01, .04]\right)$. Similarly, researcher self-confidence was linked to choosing larger prior means $\left(\beta=.14, p=.054, b=.04, \mathrm{CI}_{95}=[-.00, .08]\right)$. However, gender did not have a detectable effect on researcher confidence $(\beta=-.02, p=.796, b=-.02$, $\left.\mathrm{CI}_{95}=[-.14, .10]\right)$, and there was no mediation effect overall $\left(\beta=-.003, p=.799, b=-.00, \mathrm{CI}_{95}=\right.$ $[-.01, .00])$

Missing data handling. We did not anticipate considerable amounts of missing data in participants' responses to the research scenarios and did not pre-register how missing data would be handled when creating the composite score for the two real-research scenarios. In the main analyses, we computed the composite score only for those participants who provided answers to both real-research scenarios, treating the composite score for the rest of the participants as missing data. Missingness was then handled using FIML in all analyses. However, an alternative is to use the average of the two scenarios when both are available, and to only use one as the “composite" score when one is available. This approach retains data from more participants, potentially at the expense of quality of measurement. When we repeated our main analyses using this alternative approach, men appeared to choose higher prior means than women $(\beta=.08, p=$ $\left..070, b=.03, \mathrm{CI}_{95}=[-.00, .06]\right)$, an effect that was weak, but consistent overall with our primary analyses; once again, academic status did not significantly predict prior means $(\beta=.03, p=.487$, $\left.b=.01, \mathrm{CI}_{95}=[-.01, .03]\right)$. Coefficient estimates and $p$-values for mediation analyses were similar under the two treatments of missing data (see Table S10).

Prior standard deviations. We only pre-registered hypotheses regarding prior means, but prior standard deviations can be viewed as another way in which researchers express confidence 
PERSONAL CHARACTERISTICS AND STATISTICAL INFERENCES

(in the accuracy of their chosen effect size). When we repeated our primary analysis with this alternative dependent variable — entering gender and status into a regression predicting the average prior standard deviation set by participants in the two real research scenarios-we found no clear effects of either gender $\left(\beta=.07, p=.152, b=.01, \mathrm{CI}_{95}=[-.00, .02]\right)$ or status $(\beta=-.02$, $\left.p=.618, b=-.00, \mathrm{CI}_{95}=[-.01, .01]\right)$. However, researchers who reported higher confidence selected narrower prior distributions (path $\mathrm{b}: \beta=-.13, p=.046, b=-0.02, \mathrm{CI}_{95}=[-.03,-.00]$ ).

\section{Discussion}

Study 1 provides initial evidence that male scientists may select higher prior means than female scientists due to gender differences in researcher self-confidence. The effect emerged only when participants thought about their own research, not about hypothetical scenarios. Individuals with higher academic status also reported greater researcher self-confidence (which in turn was associated with setting higher prior means), but the overall relationship between status and prior means was not reliable.

Although we pre-registered the study, it is worth noting that we pre-registered multiple analyses with multiple independent and dependent variables. In particular, the total effect of gender on prior means for the real research composite was near the border of statistical significance $(p=.038)$, and the total effect of status was not statistically significant. Also, many participants left one or the other of the two real research scenarios blank, and we did not preregister a strategy for dealing with missing data in computing the composite score.

In light of these limitations, we conducted a replication in Study 2, focusing more narrowly on the effect of gender on the real research scenarios. Specifically, because participants' responses to each hypothetical scenario in Study 1 lacked meaningful variability (see footnote 8), we eliminated these scenarios in Study 2. We pre-registered the same analysis 
PERSONAL CHARACTERISTICS AND STATISTICAL INFERENCES

plan as in Study 1, but we predicted only an effect of gender (not status) and specified that we would handle missing data for the real-research composite by only computing the composite score for those participants who answered both real research scenarios (i.e., same as in the main analyses in Study 1). Because we were less interested in status, we broadened our inclusion criteria to include other active researchers within academia (e.g., post-docs) and we did not cap the number of graduate students who could participate, leaving us with a predominantly student sample.

\section{Study 2}

\section{Methods}

Participants. As pre-registered, we recruited active academic researchers in psychology or related fields to participate in Study $2 .{ }^{10}$ To maximize sample size, we included post-doctoral scholars as eligible participants, and did not impose a goal for the proportion of faculty members in our sample. We pre-registered a target sample size of 550, which would allow us to detect a gender difference of Cohen's $d=.25$ at above $80 \%$ power. We also pre-registered a plan to analyze Study 2 as an independent sample as long as a minimum sample size of 400 was met, which would correspond to approximately $70 \%$ power at $d=.25$.

Four hundred and fifty-nine eligible participants completed the study. Five participants did not identify as either male or female and were thus not included in our analyses, as in Study 1. Consistent with our pre-registration, we excluded an additional 43 participants who scored $60 \%$ or lower on our comprehension quiz and 4 participants who selected prior means greater than $2 .{ }^{11}$ As shown in Table 5, our sample was $61 \%$ female and 39\% male. The sample consisted

\footnotetext{
${ }^{10}$ Pre-registration available at https://osf.io/9r8ap/?view_only=7a7a77fdf82044b9bbc236377c9f09f5

${ }^{11}$ Two participants, one female and one male, selected prior means of exactly 2 on both scenarios. In technical adherence to our pre-registration, we did not exclude these participants. On an exploratory basis, we conducted the
} 
PERSONAL CHARACTERISTICS AND STATISTICAL INFERENCES

almost entirely of graduate students (88\%), with only 24 non-tenured faculty and 33 tenured faculty. Approximately half of the participants came from the social and personality area.

Procedure. The procedure of Study 2 was largely identical to that of Study 1, with two exceptions. Firstly, we only presented the two real-research scenarios (and no hypothetical scenarios) to the participants. Additionally, if participants responded that they were unable to think of a study for the second real-research scenario, they were presented with a prompt urging them to take some extra time to do so. This prompt was added in an attempt to reduce the amount of missing data in responses to the real research scenarios.

Researcher confidence scale. Based on the results of Study 1, we pre-registered using the trimmed 14-item scale to measure researcher self-confidence (although we included all 16 original items in the survey to facilitate ongoing scale validation work). Of the 459 participants who met our inclusion criteria, 29 participants had some missing data on the researcher confidence scale. One participant skipped all 14 items. As before, a 1-factor model was fit to all available data $(N=458)$ using lavaan 0.6-3 (Rosseel, 2012). Although the model again had a mediocre fit, $\chi^{2}(77)=377.95, p<.001, \mathrm{CFI}=.778, \mathrm{RMSEA}=.092, \mathrm{SRMR}=.064$, all items had reasonably high standardized loadings, between .33 and .65 . The scale had acceptable reliability $(\alpha=.81)$. A composite score was produced for each of the 458 participants based on the average of all available items for that participant to create a composite researcher self-confidence scale.

\section{Results}

\section{Pre-registered analyses.}

Predicting prior means from gender and academic status. As pre-registered, we computed the composite prior mean for each participant by taking the average of their prior 
PERSONAL CHARACTERISTICS AND STATISTICAL INFERENCES

means on both scenarios using the same approach as Study $1 .{ }^{12}$ Academic status was coded as in Study 1; post-doctoral students were grouped with graduate students. We entered gender and academic status into a regression predicting prior means. Men selected slightly higher prior means than women (for men, $M=0.45, S D=0.22$; for women, $M=0.43, S D=0.20$; illustrated in Figure 5), although this effect was not significant $\left(\beta=.06, p=.191, b=.03, \mathrm{CI}_{95}=[-.01\right.$, $.07])$, and there was no significant effect of academic status $\left(\beta=.01, p=.829, b=.00, \mathrm{CI}_{95}=[-\right.$ $.02, .03])$.

Researcher confidence as a mediator. As pre-registered, we examined whether the relationship between gender and prior means was mediated by researcher confidence. As shown in Figure 6 and the Study 2 section of Table 3, men reported trivially greater confidence than women (path a: $\left.\beta=.03, p=.541, b=.03, \mathrm{CI}_{95}=[-.06, .11]\right)$. More confident participants chose marginally higher prior means (path $\mathrm{b}: \beta=.10, p=.071, b=.05, \mathrm{CI}_{95}=[-.00, .10]$ ). The combined indirect path was not significant $\left(\beta=.00, p=.575, b=.00, \mathrm{CI}_{95}=[-.00, .01]\right)$, failing to provide evidence of mediation.

\section{Exploratory Analyses.}

Subgroup analysis: Social/personality psychologists. To reduce the heterogeneity of our overall sample, which included researchers across six areas of psychology, we repeated our primary regression analysis for social/personality psychologists, who comprised our largest subsample $(N=229)$. In this subgroup, men selected higher prior means than women $(\beta=.18, p=$ $\left..016, b=.06, \mathrm{CI}_{95}=[.01, .10]\right)$, and again there was no effect of status $(\beta=.05, p=.413, b=.01$, $\left.\mathrm{CI}_{95}=[-.02, .05]\right)$. However, the effect of gender on prior means was not mediated by researcher confidence (see Table S9).

\footnotetext{
${ }^{12}$ Missing data were handled in the same way as Study 1 . Twenty-three out of the 459 participants (5\%) did not provide an answer to at least one of the real-research scenarios, resulting in a missing composite score.
} 
PERSONAL CHARACTERISTICS AND STATISTICAL INFERENCES

Prior standard deviations. As in Study 1, we treated the average prior standard deviation as an alternative dependent variable, entering gender and status into a regression predicting this variable. Again, we found no strong effects of gender $\left(\beta=.08, p=.115, b=.01, \mathrm{CI}_{95}=[-.00\right.$, $.02])$ or status $\left(\beta=-.02, p=.589, b=-.00, \mathrm{CI}_{95}=[-.01, .01]\right)$. Consistent with Study 1 , higher self-confidence was linked to choosing slightly narrower priors, although the effect did not reach significance $\left(\beta=-.09, p=.071, b=-.01, \mathrm{CI}_{95}=[-.03, .001]\right)$.

\section{Combined Results Across Studies 1 \& 2 (Exploratory)}

Although we pre-registered a target sample size of 550 participants for Study 2, we fell short of this goal, primarily due to budget constraints. We pre-registered a plan to analyze the data for Study 2 separately as long as we obtained a minimum sample size of at least 400, which we met $(N=459)$. Because Study 2 was somewhat underpowered, we also present our key analyses combining across Studies 1 and 2 below $(N=944)$, although these analyses should be treated as exploratory.

\section{Predicting Prior Means from Gender and Academic Status}

As before, we entered gender and status into a regression predicting the composite prior means. Men selected significantly higher prior means $(M=0.44, S D=0.20)$ than women $(M=$ $.41, S D=.18), \beta=.08, p=.022, b=.03, \mathrm{CI}_{95}=[.00, .06]$; illustrated in Figure 7. There was no effect of status $\left(\beta=-.02, p=.591, b=-.00, \mathrm{CI}_{95}=[-.02, .01]\right)$.

\section{Researcher Confidence as a Mediator}

Repeating our mediation analysis (see Figure 8), we found that men reported higher levels of confidence than women $\left(\beta=.08, p=.014, b=.08, \mathrm{CI}_{95}=[.02, .14]\right)$, and more confident researchers chose higher prior means $\left(\beta=.12, p=.002, b=.05, \mathrm{CI}_{95}=[.02, .08]\right)$. The indirect effect of gender on prior means via confidence was marginally significant $(\beta=.01, p=$ 
PERSONAL CHARACTERISTICS AND STATISTICAL INFERENCES

$\left..061, b=.00, \mathrm{CI}_{95}=[-.00, .01]\right)$. After taking this indirect effect into account, there was still a significant direct effect of gender on prior means $\left(\beta=.07, p=.037, b=.03, \mathrm{CI}_{95}=[.00, .05]\right)$, suggesting that gender differences in researcher confidence could only partially explain why men chose higher prior means than women.

\section{Previous experience in statistics}

Our most puzzling finding was that status had no effect on prior means, even though status was strongly related to increased confidence. One possibility is that while participants with higher academic status would have selected higher prior means due to their high confidence, they also had more statistics training, which might be linked to more realistic expectations of effect sizes. If this were the case, the competing effects of confidence and statistical experience would cancel each other out, resulting in an overall null effect of status on prior means. To explore this idea, we ran a full mediation model predicting prior means from status, using two correlated mediators: confidence and experience in statistics (see Figure 9). Applying the same model specifications as our other mediation analyses, we found that status predicted confidence (path a: $\left.\beta=.26, p<.001, b=.17, \mathrm{CI}_{95}=[.13, .21]\right)$, and confidence in turn predicted prior means (path $\mathrm{b}$ : $\beta=.14, p<.001, b=.06, \mathrm{CI}_{95}=[.02, .09]$. Based on this combined pathway alone (path $\mathrm{a}^{*} \mathrm{~b}: \beta=$ $\left..04, p=.002, b=.01, \mathrm{CI}_{95}=[.00, .02]\right)$, we would infer that participants with higher academic status chose higher prior means due to their higher researcher self-confidence. However, participants with higher academic status also reported more statistical experience (path c: $\beta=.22$, $\left.p<.001, b=.22, \mathrm{CI}_{95}=[.15, .28]\right)$, and those with more statistical experience chose marginally lower prior means (path $\mathrm{d}: \beta=-.07, p=.055, b=-.02, \mathrm{CI}_{95}=[-.04, .00]$ ) when the other pathways are controlled for (path $\mathrm{c}^{*} \mathrm{~d}: \beta=-.02, p=.068, b=-.00, \mathrm{CI}_{95}=[-.01, .00]$ ). These exploratory findings tentatively suggest that researchers with higher status tend to be more self- 
PERSONAL CHARACTERISTICS AND STATISTICAL INFERENCES

confident, which is linked to selecting larger prior means, but they also tend to have more statistical expertise, which is linked to selecting smaller prior means—and these two effects appear to counteract one another.

\section{General Discussion}

In the present research, we conducted the first empirical investigation of how psychological scientists engage in Bayesian reasoning. Across studies, our most robust finding was that more self-confident researchers selected higher prior means. We also observed mixed evidence that men may choose larger prior means than women; this effect was significant in Study 1, but not Study 2. Combining across both studies with over 900 active researchers in psychology, we found that men selected higher prior means than women when asked to think about their own research studies; this effect (approximately $d=.16$ ) was smaller than the modal effect size across social psychology ( $d=.36$; Richard, Bond, \& Stokes-Zoota, 2003). Across studies, we found suggestive evidence that the effect of gender on prior means was mediated by researcher self-confidence; however, our mediation analyses did not reach significance in Study 2, and thus should be interpreted with considerable caution. In addition, we saw that those with higher academic status reported greater self-confidence—-but not surprisingly, people with higher status also had more statistical experience. While higher self-confidence was linked to choosing larger prior means, statistical experience was linked to choosing smaller prior means, which might help to explain why we did not observe an overall effect of status on prior means. Taken together, our findings suggest that more self-confident researchers are inclined to select larger prior means, and demographic variables such as gender and status may shape researcher selfconfidence - but demographic variables may also influence prior means in other ways. 
PERSONAL CHARACTERISTICS AND STATISTICAL INFERENCES

Although we only pre-registered hypotheses regarding prior means, prior standard deviations may provide an alternative expression of confidence. While we did not find clear effects of gender or status on prior standard deviations in either study, we found that more selfconfident researchers selected significantly narrower standard deviations in Study 1. This effect was very similar in size (though only marginally significant) in Study 2. Thus, the current findings point to the possibility that researcher self-confidence may be linked to the choice of both components of the prior distribution, though we would speculate that these effects may be easier to detect in samples with higher levels of statistical expertise. Indeed, a critical difference between Study 1 and Study 2 was that participants in Study 2 (vs. Study 1) reported a lower level of statistical expertise and included a much lower proportion of faculty (12\% vs. $45 \%)$. This difference might help to explain why we observed clearer effects — almost across the board —in Study 1 relative to Study 2 .

\section{Limitations and Future Research}

A central limitation of the present work is that gender, confidence, and status were measured rather than manipulated. In particular, although we theorized that gender differences in confidence should lead male (vs. female) researchers to set higher prior means, this pattern could also have emerged if men simply choose to study effects that are genuinely larger than the effects women choose to study. Indeed, it is possible that obtaining large effects increases researcher self-confidence, helping to account for the gender difference in self-confidence we observed. This problem is compounded by the fact that we drew participants from across diverse areas of psychology; it is possible that men might be overrepresented in areas of psychology in which larger effects are typically observed. However, we found a consistent effect of gender on prior means within our largest subgroup (Social/Personality Psychology), suggesting that our observed 
PERSONAL CHARACTERISTICS AND STATISTICAL INFERENCES

effects cannot be easily explained by different gender representations across different areas of psychology. Of course, actual effect sizes are likely to differ within sub-areas of psychology, and men might simply be more drawn to studying larger effect sizes. To circumvent this seemingly intractable issue, it would be interesting to examine registered reports in psychology; if our theoretical perspective is correct, then all-male author teams may predict larger effect sizes than all-female author teams. Because the authors' predicted effect sizes could be compared with the actual effect sizes that emerged, providing a benchmark for accuracy, this approach would make it possible to assess whether men are overconfident on average.

Constraints on generalizability. Because our sample consisted entirely of psychologists in North America, special caution is warranted in generalizing these findings to other cultural contexts. Indeed, most previous work on gender differences in confidence regarding math and science has been conducted in North America. Moreover, only a handful of participants with a gender identity other than male or female completed the study, precluding any meaningful conclusions about the relationship between confidence and setting Bayesian prior means for people across the gender continuum (see Connell, 2012). Future researchers should strive to utilize well-powered samples of people with diverse gender identities and heed recommendations to operationalize gender using multidimensional, continuous measures (see Ansara \& Hegarty, 2014; Hyde et al., 2019; Lindqvist et al., 2020). It is also important to note that researchers voluntarily completed our study, and individuals with some interest in Bayesian statistics may have been particularly likely to participate. From our theoretical perspective, the observed gender differences should also emerge among researchers in other areas of science, but it would be important to test this extension empirically. 
PERSONAL CHARACTERISTICS AND STATISTICAL INFERENCES

Our researcher confidence scale could potentially be used to test the generalizability of gender differences in confidence among researchers in diverse fields of science and regions of the world. Although the scale will require further development and validation for broader use, the present research provides initial evidence for construct validity, in that our scale captures expected group differences in researcher self-confidence (e.g., individuals with higher academic status report greater self-confidence ${ }^{13}$ ). As part of the present research, we were able to collect pilot data $(N=72)$ on the initial version of the scale, which led to the improvements in item wording, but this initial sample size was insufficient for full psychometric validation. While the developed scale had good internal consistency and all 14 items had reasonably high standardized loadings in both studies, the 1-factor model had poor fit to the data. We are currently conducting additional scale validation work to establish whether the scale encompasses more than one factor, in order to facilitate future research using this scale.

\section{Implications}

Keeping in mind the important limitations highlighted above, our studies point to the conclusion that more self-confident researchers may select larger prior means and narrower prior standard deviations than their humbler colleagues. As a result, more self-confident researchers are likely to obtain larger Bayes Factors, all else being equal. Although we focused on gender and status as predictors of self-confidence, we would theorize that any variable that increases confidence — such as being at a prestigious university—might also have downstream consequences for the priors that researchers select. Specifically, these extraneous variables may

${ }^{13}$ Academic status was, of course, strongly correlated with age $(r=.73$ for the combined sample across studies). It would be interesting to examine whether people who obtained their PhD's during or after the replication crisis are more inclined toward humility than people who received their training during earlier periods. 
PERSONAL CHARACTERISTICS AND STATISTICAL INFERENCES

increase researchers' priors without altering the effect sizes they are studying, producing overconfidence.

While researchers routinely make many subjective decisions even when using traditional statistical methods, a unique feature of Bayesian hypothesis testing is that these subjective judgements are explicitly incorporated into the computations and directly impact the size of the resulting statistic (i.e., the Bayes Factor). This feature of Bayesian analyses makes it relatively easy to study the impact of subjective judgements on statistical conclusions. That said, the present research carries implications for statistical inference more broadly, whether Bayesian or frequentist. For example, if men anticipate higher effect sizes than women, men may be more likely to make bold predictions in grant applications (which often require effect size estimates) or to underpower their studies. In fact, the implications for users of frequentist statistics may be more insidious because researchers' subjective judgements are less transparent, but may still shape research decisions. 
PERSONAL CHARACTERISTICS AND STATISTICAL INFERENCES

\section{References}

Aknin, L. B., Dunn, E. W., Proulx, J., Lok, I., \& Norton, M. I. (2020). Does spending money on others promote happiness?: A registered replication report. Journal of Personality and Social Psychology. Advance online publication. https://doi.org/10.1037/pspa0000191

Ansara, Y. G., \& Hegarty, P. (2014). Methodologies of misgendering: Recommendations for reducing cisgenderism in psychological research. Feminism \& Psychology, 24(2), 259270. doi:10.1177/0959353514526217

Beyer, S., \& Bowden, E.M. (1997). Gender Differences in Self-Perceptions: Convergent Evidence from Three Measures of Accuracy and Bias. Personality and Social Psychology Bulletin, 23, 157-172. doi.org/10.1177/0146167297232005

Byrnes, J. P., Miller, D. C., \& Schafer, W. D. (1999). Gender differences in risk taking: A metaanalysis. Psychological Bulletin, 125, 367-383. doi:10.1037/0033-2909.125.3.367

Charness, G., Gneezy, U., (2012). Strong evidence for gender differences in risk-taking. Journal of Economic Behavior and Organization, 83(1), 50-58.

\section{doi.org/10.1016/j.jebo.2011.06.007}

Connell, R. (2012). Gender, health and theory: Conceptualizing the issue, in local and world perspective. Social Science \& Medicine, 74(11), 1675-1683.

doi:10.1016/j.socscimed.2011.06.006

Dienes, Z. (2011). Bayesian versus orthodox statistics: Which side are you on? Perspectives on Psychological Science, 6(3), 274-290. doi:10.1177/1745691611406920

Dienes, Z. (2014). Using bayes to get the most out of non-significant results. Frontiers in Psychology, 5, 781. doi:10.3389/fpsyg.2014.00781 
PERSONAL CHARACTERISTICS AND STATISTICAL INFERENCES

Dunning, D. (2005). Self-insight: Roadblocks and detours on the path to knowing thyself. New York: Psychology Press.

Ehrlinger, J., \& Dunning, D. (2003). How chronic self-views influence (and potentially mislead) estimates of performance. Journal of Personality and Social Psychology, 84(1), 5-17. doi:10.1037/0022-3514.84.1.5

Ehrlinger, J., Plant, A. E., Hartwig, M., Vossen, J. J., Columb, C. J., \& Brewer, L. (2017). Do women hold more exaggerated perceptions of CS and engineering prototypes than men and, consequently, show less interest in these fields? Sex Roles, 78(1-2), 40-51. doi:10.1007/s11199-017-0763-X

Ellis, J., Fosdick, B.K., \& Rasmussen, C. (2016). Women 1.5x more likely to leave STEM pipeline after calculus compared to men: Lack of mathematical confidence a potential culprit. PloS One, 11(7), e0157447. https://doi.org/10.1371/journal .pone.0157447

Else-Quest, N. M., Hyde, J. S., \& Linn, M. C. (2010). Cross-national patterns of gender differences in mathematics: A meta-analysis. Psychological Bulletin, 136(1), 103-127. doi: $10.1037 / \mathrm{a} 0018053$

Huang, C. (2013). Gender differences in academic self-efficacy: a meta-analysis. European Journal of Psychology of Education, 28(1). 1-35. doi:10.1007/s10212-011-0097-y. 
PERSONAL CHARACTERISTICS AND STATISTICAL INFERENCES

Hyde, J. S., Bigler, R. S., Joel, D., Tate, C. C., \& van Anders, S. M. (2019). The future of sex and gender in psychology: Five challenges to the gender binary. The American Psychologist, 74(2), 171-193. doi:10.1037/amp0000307

Inbar, Y. \& Inzlicht, M. (Hosts). (2020, March 4). Diversity, death, and (Cohen's d). In Y. Inbar \& M. Inzlicht (Executive Producers). Two psychologists four beers [Audio podcast] (Episode 40). Retrieved from https://fourbeers.fireside.fm/40.

Instone, D., Major, B., \& Bunker, B.B. (1983). Gender, self confidence, and social influence strategies: An organizational simulation. Journal of Personality and Social Psychology, 44(2), 322-333. doi:10.1037/0022-3514.44.2.322

Lerchenmueller, M. J., Sorenson, O., and Jena, A. B. (2019). Gender differences in how scientists present the importance of their research: Observational study. British Medical Journal, 367, 16573. doi:10.1136/BMJ.L6573

Lindqvist, A., Sendén, M. G., Renström, E. A. (2020). What is gender, anyway: A review of the options for operationalising gender. Psychology \& Sexuality,1-13. https://doi.org/10.1080/19419899.2020.1729844

Micari, M., Pazor, P., \& Hartmann, M. J. Z. (2007). A matter of confidence: Gender differences in attitudes toward engaging in lab and course work in undergraduate engineering. Journal of Women and Minorities in Science and Engineering, 13(3), 279-293. https://doi.org/10.1615/JWomenMinorScienEng.v13.i3.50

Min, D. \& Kim, J. (2013). Is power powerful? Power, confidence, and goal pursuit. International Journal of Research in Marketing, 30, 265-275. doi:10.1016/j.ijresmar.2012.12.001.

Open Science Collaboration (2015). Estimating the reproducibility of psychological science. Science, 349(6251). doi:10.1126/science.aac4716 
PERSONAL CHARACTERISTICS AND STATISTICAL INFERENCES

Reisner, S. L., Conron, K. J., Scout, Baker, K., Herman, J. L., Lombardi, E., . . Matthews, A. K. (2015). "Counting" transgender and gender-nonconforming adults in health research. Transgender Studies Quarterly, 2(1), 34-57. doi:10.1215/23289252-2848877

Richard, F. D., Bond, C. F., \& Stokes-Zoota, J. J. (2003). One hundred years of social psychology quantitatively described. Review of General Psychology, 7(4), 331-363. doi:10.1037/1089-2680.7.4.331

Rosseel, Y. (2012). lavaan: An R package for structural equation modeling. Journal of Statistical Software, 48(2), 1-36. Retrieved from http://www.jstatsoft.org/v48/i02/.

Rouder, J. N., Speckman, P. L., Sun, D., Morey, R. D., and Iverson, G. (2009). Bayesian t tests for accepting and rejecting the null hypothesis. Psychonomic Bulletin \& Review, 16, 225 237. doi:10.3758/PBR.16.2.225

See, K. E., Morrison, E. W., Rothman, N. B. \& Soll, J. B. (2011). The detrimental effects of power on confidence, advice taking, and accuracy. Organizational Behavior and Human Decision Processes, 116, 272-285. doi:10.1016/j.obhdp.2011.07.006

Simmons, J. P., Nelson, L. N., \& Simonsohn, U. (2011). False-positive psychology: Undisclosed flexibility in data collection and analysis allows presenting anything as significant. Psychological Science, 22, 1359-1366. doi: 10.1177/0956797611417632

Simonsohn, U., Nelson, L. D., \& Simmons, J. P. (2014a). P-curve: A key to the filedrawer. Journal of Experimental Psychology. General, 143(2), 534-547. doi: $10.1037 / \mathrm{a} 0033242$

Simonsohn, U., Nelson, L. D., \& Simmons, J. P. (2014b). P-curve and effect size: Correcting for publication bias using only significant results. Perspectives on Psychological Science, 9(6), 666-681. doi:10.1177/1745691614553988 
PERSONAL CHARACTERISTICS AND STATISTICAL INFERENCES

Slaney, K. L. (2001). On empirical realism and the defining of theoretical terms. Journal of Theoretical and Philosophical Psychology, 21(2), 132-152. doi:10.1037/h0091202

Stankov, L., \& Lee, Y.-P. (2014). Overconfidence across world regions. Journal of CrossCultural Psychology, 45(5), 821-837. doi.org/10.1177/0022022114527345 
PERSONAL CHARACTERISTICS AND STATISTICAL INFERENCES

Table 1. Summary of sample demographics (Study 1).

\begin{tabular}{|c|c|c|c|c|}
\hline Study 1 & $\begin{array}{c}\text { Total } \\
(N=485)\end{array}$ & $\begin{array}{l}\text { Graduate } \\
\text { Students } \\
(N=266)\end{array}$ & $\begin{array}{c}\text { Non-Tenured } \\
\text { Faculty } \\
(N=129)\end{array}$ & $\begin{array}{l}\text { Tenured } \\
\text { Faculty } \\
(N=90)\end{array}$ \\
\hline Female & 284 & 164 & 75 & 45 \\
\hline Male & 201 & 102 & 54 & 45 \\
\hline Social/Personality & 203 & 101 & 63 & 39 \\
\hline Cognitive & 62 & 36 & 14 & 12 \\
\hline Clinical & 84 & 68 & 9 & 7 \\
\hline Health & 10 & 5 & 2 & 3 \\
\hline $\begin{array}{l}\text { Biological/Behavioural } \\
\text { Neuroscience }\end{array}$ & 20 & 16 & 2 & 2 \\
\hline Developmental & 72 & 22 & 27 & 23 \\
\hline Other (quant, $\mathrm{I} / \mathrm{O}$, etc.) & 34 & 18 & 12 & 4 \\
\hline Canada & 51 & 23 & 13 & 15 \\
\hline \multirow[t]{2}{*}{ United States } & 434 & 243 & 116 & 75 \\
\hline & $M(S D)$ & $M(S D)$ & $M(S D)$ & $M(S D)$ \\
\hline Experience with Statistics & $2.79(.70)$ & $2.61(.61)$ & $3.03(.72)$ & $3.00(.75)$ \\
\hline $\begin{array}{c}\text { Familiarity with Bayesian } \\
\text { Statistics }\end{array}$ & $1.65(.72)$ & $1.58(.66)$ & $1.73(.78)$ & $1.73(.80)$ \\
\hline
\end{tabular}

Note: Experience with Statistics: 1 = equivalent to undergraduate courses only; 2 = equivalent to $1-2$ graduate courses; 3 = equivalent to 3 or more graduate courses; $4=$ even better (e.g., you have a degree in quantitative psychology; you have spent years teaching yourself advanced statistics for use in your research; you teach grad statistics courses; etc.). Familiarity with Bayesian Statistics: $1=$ "Not at all (may have heard whispers in the hallway)"; 2 = "A bit (equivalent to a few lectures/pre-conference workshop/1-2 articles/or you have used it in your research with the help of a consultant)"; 3 = "A fair amount (equivalent to a course or so)"; 4 = "Very (more than one course and/or you use it in your research); 5 = Very very (You teach it! You write about it!)." Frequency distributions of these variables are available in Supplemental Material Tables S1 and S2. 
PERSONAL CHARACTERISTICS AND STATISTICAL INFERENCES

Table 2. Standardized effects of gender and academic status on prior means for each Hypothetical Scenario (HS), Real Research Scenario (RRS), and the Real Research composite (Study 1).

\begin{tabular}{ccccccc}
\hline Study 1 & HS 1 & HS 2 & HS3 & RRS 1 & RRS 2 & RRS Composite \\
\hline \multirow{2}{*}{ Gender } & $\beta=-.02$, & $\beta=-.02$, & $\beta=. .08$, & $\beta=.09$, & $\beta=.07$, & $\beta=.10$, \\
& $p=.722$ & $p=.644$ & $p=.062$ & $p=.066$ & $p=.173$ & $\boldsymbol{p}=. \mathbf{0 3 8}$ \\
Academic & $\beta=-.04$, & $\beta=.000$, & $\beta=-.03$, & $\beta=.06$, & $\beta=-.04$, & $\beta=-.001$, \\
Status & $p=.392$ & $p=.998$ & $p=.442$ & $p=.265$ & $p=.446$ & $p=.984$ \\
\hline
\end{tabular}

Note. The reported values are $z$-tests because the regressions were fit as path analyses in lavaan. Given a large sample, this distinction does not have any practical consequences. The independent variables (gender and academic status) were essentially uncorrelated (point-biserial correlation $r_{p b}=0.11, \chi^{2}(2)=$ $3.67, p=.16$ ), so the beta coefficients are very close to bivariate correlations. $P$-values smaller than .05 are bolded. HS: Hypothetical Scenario. RRS: Real Research Scenario. 
PERSONAL CHARACTERISTICS AND STATISTICAL INFERENCES

Table 3. Mediation models of gender effects $(\beta \mathrm{s})$ on the prior means with confidence (trimmed scale) as a mediator for each Real Research Scenario (RRS) and the Real Research composite.

\begin{tabular}{|c|c|c|c|}
\hline $\begin{array}{c}\text { Model Paths } \\
\text { Study } 1\end{array}$ & RRS 1 & RRS 2 & RRS Composite \\
\hline $\begin{array}{l}\text { Gender on self-confidence } \\
\text { (path a) }\end{array}$ & $.12(p=.006)$ & $.12(p=.006)$ & $.12(p=.006)$ \\
\hline $\begin{array}{l}\text { Self-confidence on prior } \\
\text { means (path b) }\end{array}$ & $.19(p<.001)$ & $.08(p=.143)$ & $.17(p<.001)$ \\
\hline Indirect Path $(\mathrm{a} \times \mathrm{b})$ & $.02(p=.024)$ & $.01(p=.179)$ & $.02(p=.031)$ \\
\hline $\begin{array}{l}\text { Direct Path: Gender on } \\
\text { prior means (path c) }\end{array}$ & $.07(p=.117)$ & $.06(p=.247)$ & $.09(p=.069)$ \\
\hline Total & $.10(p=.043)$ & $.07(p=.184)$ & $.11(p=.029)$ \\
\hline Study 2 & RRS 1 & RRS 2 & RRS Composite \\
\hline $\begin{array}{l}\text { Gender on self-confidence } \\
\text { (path a) }\end{array}$ & $.03(p=.623)$ & $.03(p=.545)$ & $.03(p=.541)$ \\
\hline $\begin{array}{l}\text { Self-confidence on prior } \\
\text { means (path b) }\end{array}$ & $.13(p=.009)$ & $.54(p=.385)$ & $.10(p=.071)$ \\
\hline Indirect Path $(\mathrm{a} \times \mathrm{b})$ & $.00(p=.554)$ & $.00(p=.630)$ & $.00(p=.575)$ \\
\hline $\begin{array}{l}\text { Direct Path: Gender on } \\
\text { prior means (path c) }\end{array}$ & $.11(p=\mathbf{. 0 3 3})$ & $-.02(p=.707)$ & $.06(p=.212)$ \\
\hline Total & $.11(p=.029)$ & $-.02(p=.730)$ & $.06(p=.197)$ \\
\hline Combined (Study 1 \& 2) & RRS 1 & RRS 2 & RRS Composite \\
\hline $\begin{array}{l}\text { Gender on self-confidence } \\
\text { (path a) }\end{array}$ & $.08(p=. \mathbf{0 1 3})$ & $.08(p=.014)$ & $.08(p=.014)$ \\
\hline $\begin{array}{l}\text { Self-confidence on prior } \\
\text { means (path b) }\end{array}$ & $.14(p<.001)$ & $.05(p=.191)$ & $.12(p=. \mathbf{0 0 2})$ \\
\hline Indirect Path $(\mathrm{a} \times \mathrm{b})$ & $.01(p=.038)$ & $.00(p=.251)$ & $.01(p=.061)$ \\
\hline $\begin{array}{l}\text { Direct Path: Gender on } \\
\text { prior means (path c) }\end{array}$ & $.09(p=.009)$ & $.01(p=.686)$ & $.07(p=. \mathbf{0 3 7})$ \\
\hline Total & $.10(p=.004)$ & $.02(p=.608)$ & $.08(p=\mathbf{. 0 2 1})$ \\
\hline
\end{tabular}

Note. Table cells give the standardized regression coefficients and the corresponding $p$-value in parentheses. $P$-values smaller than .05 are bolded. RRS: Real Research Scenario. 
PERSONAL CHARACTERISTICS AND STATISTICAL INFERENCES

Table 4. Mediation models of status effects $(\beta \mathrm{s})$ on the prior means with confidence (trimmed scale) as a mediator for each Real Research Scenario (RRS) and the Real Research composite (Study 1).

\begin{tabular}{cccc}
\hline Study 1 & RRS 1 & RRS 2 & RRS Composite \\
\hline $\begin{array}{c}\text { Status on self-confidence } \\
\text { (path a) }\end{array}$ & $.30(p<. \mathbf{0 0 1})$ & $.30(p<. \mathbf{0 0 1})$ & $.30(p<. \mathbf{0 0 1})$ \\
$\begin{array}{c}\text { Self-confidence on prior } \\
\text { means (path b) }\end{array}$ & $.20(p<. \mathbf{0 0 1})$ & $.10(p=.064)$ & $.20(p<. \mathbf{0 0 1})$ \\
$\begin{array}{c}\text { Indirect Path (a x b) } \\
\text { Direct Path: Status on prior } \\
\text { means (path c) }\end{array}$ & $.06(p<. \mathbf{0 0 1})$ & $.03(p=.077)$ & $.06(p=. \mathbf{0 0 1})$ \\
Total & $.07(p=.197)$ & $-.06(p=.222)$ & $-.05(p=.329)$ \\
\end{tabular}

Note. Table cells give the standardized regression coefficients and the corresponding $p$-value in parentheses. $P$-values smaller than .05 are bolded. RRS: Real Research Scenario. 
PERSONAL CHARACTERISTICS AND STATISTICAL INFERENCES

Table 5. Summary of sample demographics (Study 2).

\begin{tabular}{|c|c|c|c|c|}
\hline Study 2 & $\begin{array}{c}\text { Total } \\
(N=459)\end{array}$ & $\begin{array}{l}\text { Graduate } \\
\text { Students } \\
(N=402)\end{array}$ & $\begin{array}{c}\text { Non-Tenured } \\
\text { Faculty } \\
(N=24)\end{array}$ & $\begin{array}{l}\text { Tenured } \\
\text { Faculty } \\
(N=33)\end{array}$ \\
\hline Female & 281 & 246 & 16 & 19 \\
\hline Male & 178 & 156 & 8 & 14 \\
\hline Social/Personality & 229 & 199 & 12 & 18 \\
\hline Cognitive & 79 & 66 & 6 & 7 \\
\hline Clinical & 40 & 39 & 0 & 1 \\
\hline Developmental & 40 & 36 & 1 & 3 \\
\hline $\begin{array}{l}\text { Biological/Behavioural } \\
\text { Neuroscience }\end{array}$ & 22 & 20 & 1 & 1 \\
\hline Health & 19 & 17 & 1 & 1 \\
\hline Other (quant, I/O, etc.) & 30 & 25 & 3 & 2 \\
\hline Canada & 39 & 35 & 3 & 1 \\
\hline \multirow[t]{2}{*}{ United States } & 420 & 367 & 21 & 32 \\
\hline & $M(S D)$ & $M(S D)$ & $M(S D)$ & $M(S D)$ \\
\hline Experience with Statistics & $1.73(.66)$ & $1.68(.64)$ & $2.08(.72)$ & $2.03(.73)$ \\
\hline $\begin{array}{l}\text { Familiarity with Bayesian } \\
\text { Statistics }\end{array}$ & $1.68(.71)$ & $1.66(.70)$ & $1.88(.68)$ & $1.78(.86)$ \\
\hline
\end{tabular}

Note: Experience with Statistics: 1 = equivalent to undergraduate courses only; 2 = equivalent to $1-2$ graduate courses; 3 = equivalent to 3 or more graduate courses; $4=$ even better (e.g., you have a degree in quantitative psychology; you have spent years teaching yourself advanced statistics for use in your research; you teach grad statistics courses; etc.). Familiarity with Bayesian Statistics: 1 = "Not at all (may have heard whispers in the hallway)"; 2 = "A bit (equivalent to a few lectures/pre-conference workshop/1-2 articles/or you have used it in your research with the help of a consultant)"; $3=$ "A fair amount (equivalent to a course or so)"; 4 = "Very (more than one course and/or you use it in your research); 5 = Very very (You teach it! You write about it!)." Frequency distributions of these variables are available in Supplemental Material Tables S11 and S12. 

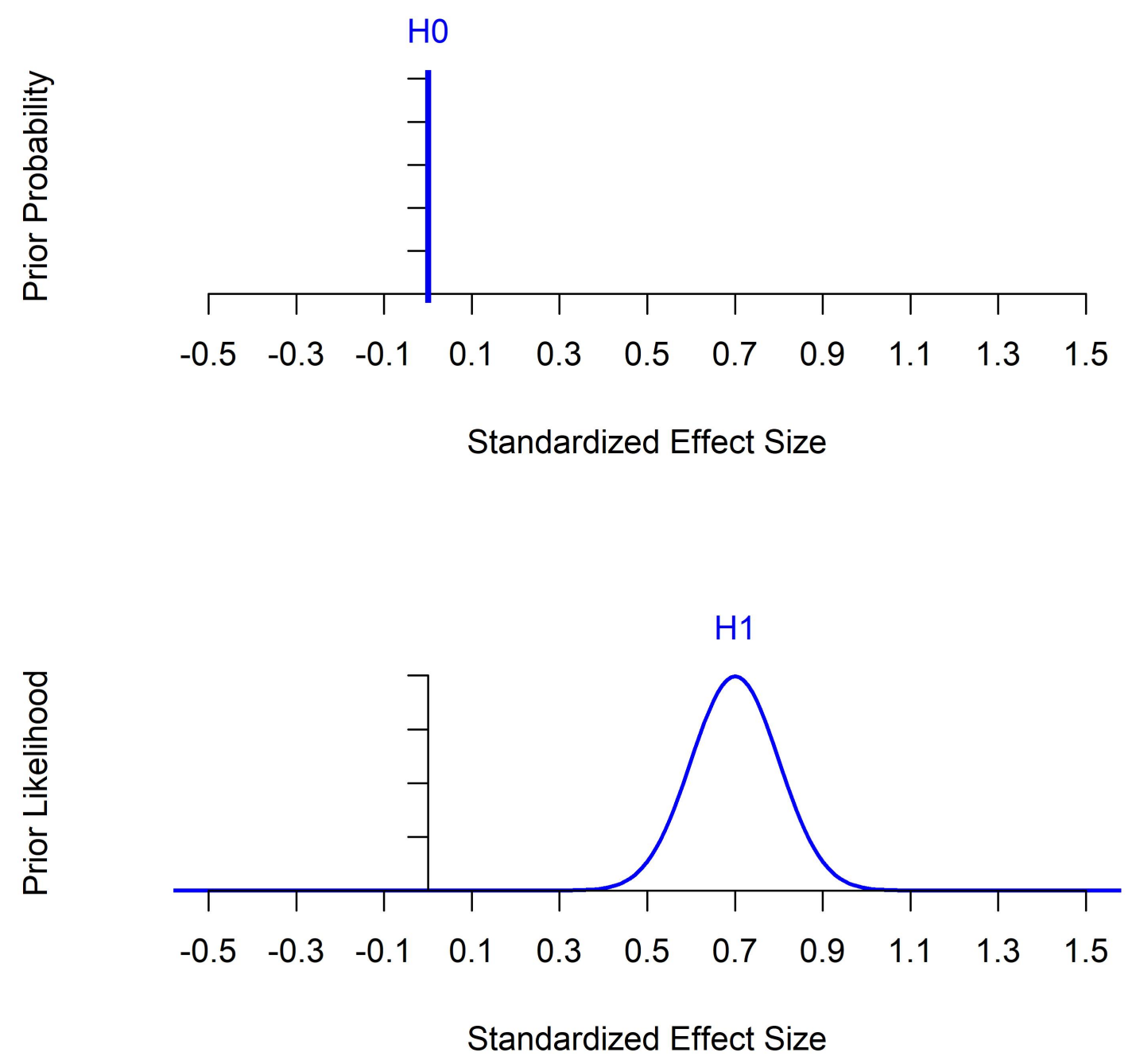

Figure 1. Illustration of the null $\left(H_{0}: \delta=0\right)$ and the alternative $\left(H_{1}: \delta \sim N\left(.7, .1^{2}\right)\right)$ hypotheses in Bayesian hypothesis testing.

Note. These hypotheses can be stated for the raw effect size (e.g., mean difference) or for the standardized effect size ( $\delta$ ), as is shown here. $\mathrm{H}_{0}: \delta=0$ is the traditional null hypothesis. In contrast, $\mathrm{H}_{1}$ specifies a probability distribution for $\delta$, called the prior distribution. In this example, the prior for $\delta$ is normal, with the prior mean of .7 and prior standard deviation of .1 (or variance of $.1^{2}$ ). By specifying such a prior, the researcher is stating that, prior to seeing any data, they are $95 \%$ confident that the true effect size falls between .5 and .9 . 
PERSONAL CHARACTERISTICS AND STATISTICAL INFERENCES
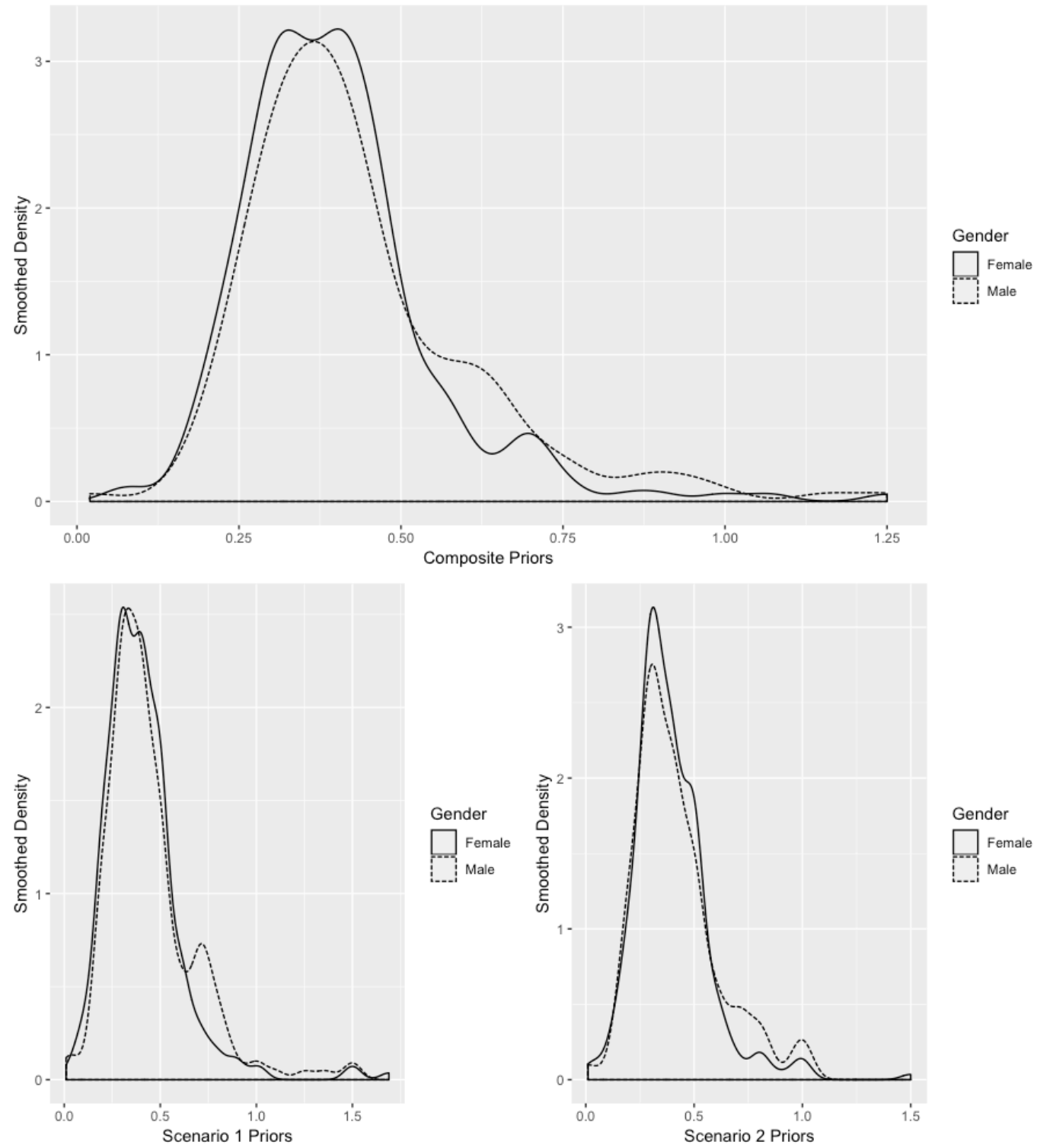

Figure 2. The density distributions of prior means chosen by women and men in Study 1. Note. The densities were smoothed using Gaussian kernel density estimation. 
PERSONAL CHARACTERISTICS AND STATISTICAL INFERENCES

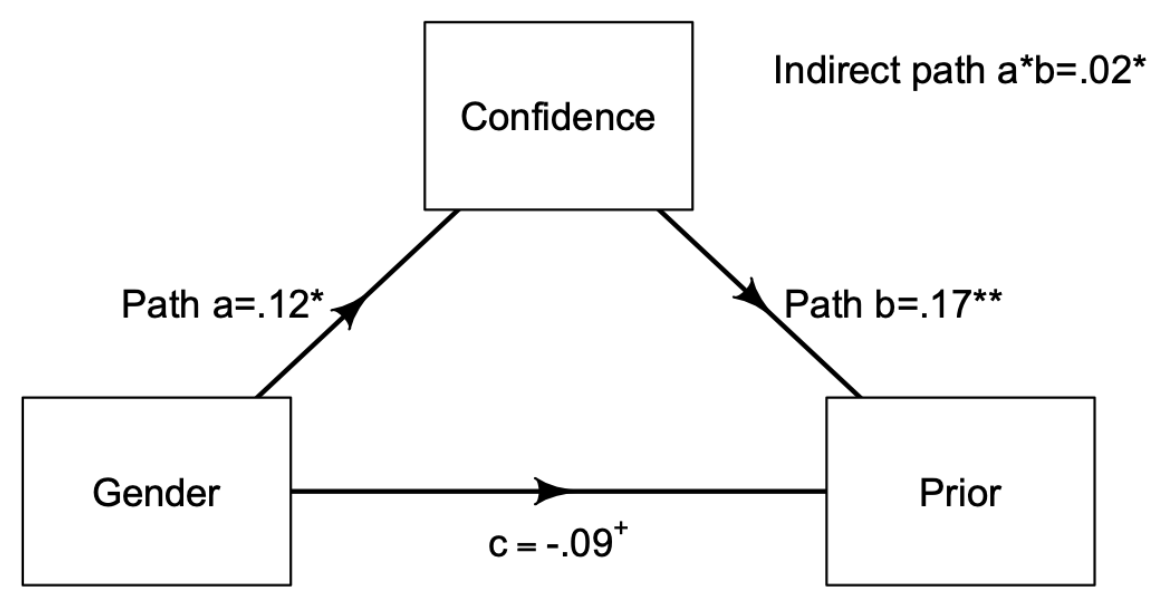

Total effect $=.11^{*}$

Figure 3. Path diagram of gender on the Real Research composite prior mean mediated by selfconfidence in Study 1.

Note. ${ }^{* *}: p<.001 ;{ }^{*} p<.05 ;^{+}: p<.10$; otherwise: $p \geq .10$. 
PERSONAL CHARACTERISTICS AND STATISTICAL INFERENCES

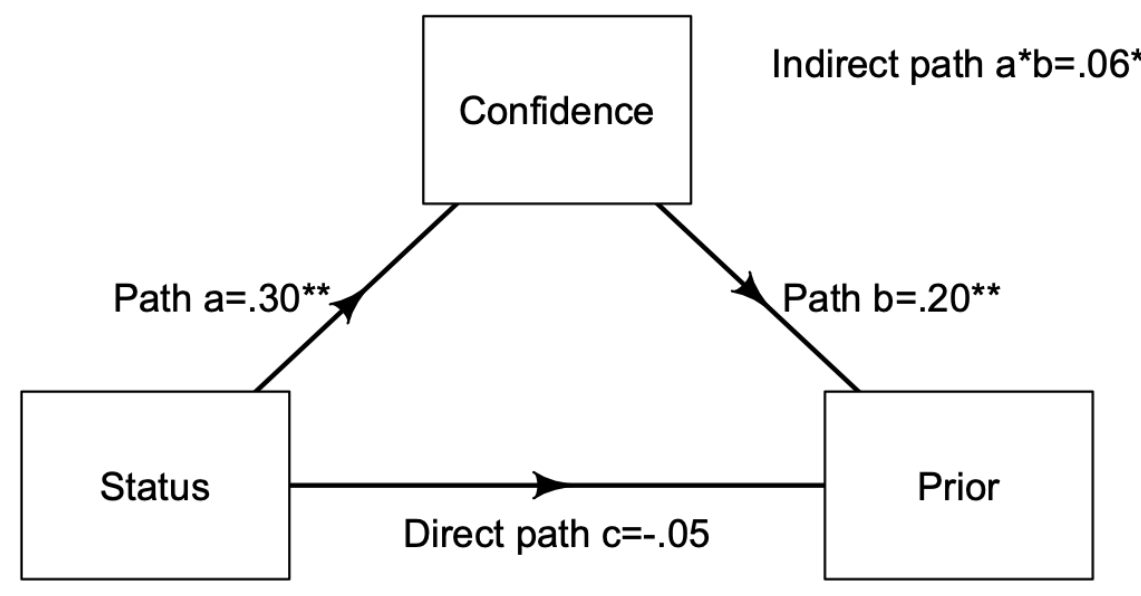

Total effect $=.01$

Figure 4. Path diagram of academic status on the Real Research composite prior mean mediated by self-confidence in Study 1.

Note. ${ }^{* *}: p<.001 ;{ }^{*} p<.05 ;^{+}: p<.10 ;$ otherwise: $p \geq .10$. 
PERSONAL CHARACTERISTICS AND STATISTICAL INFERENCES
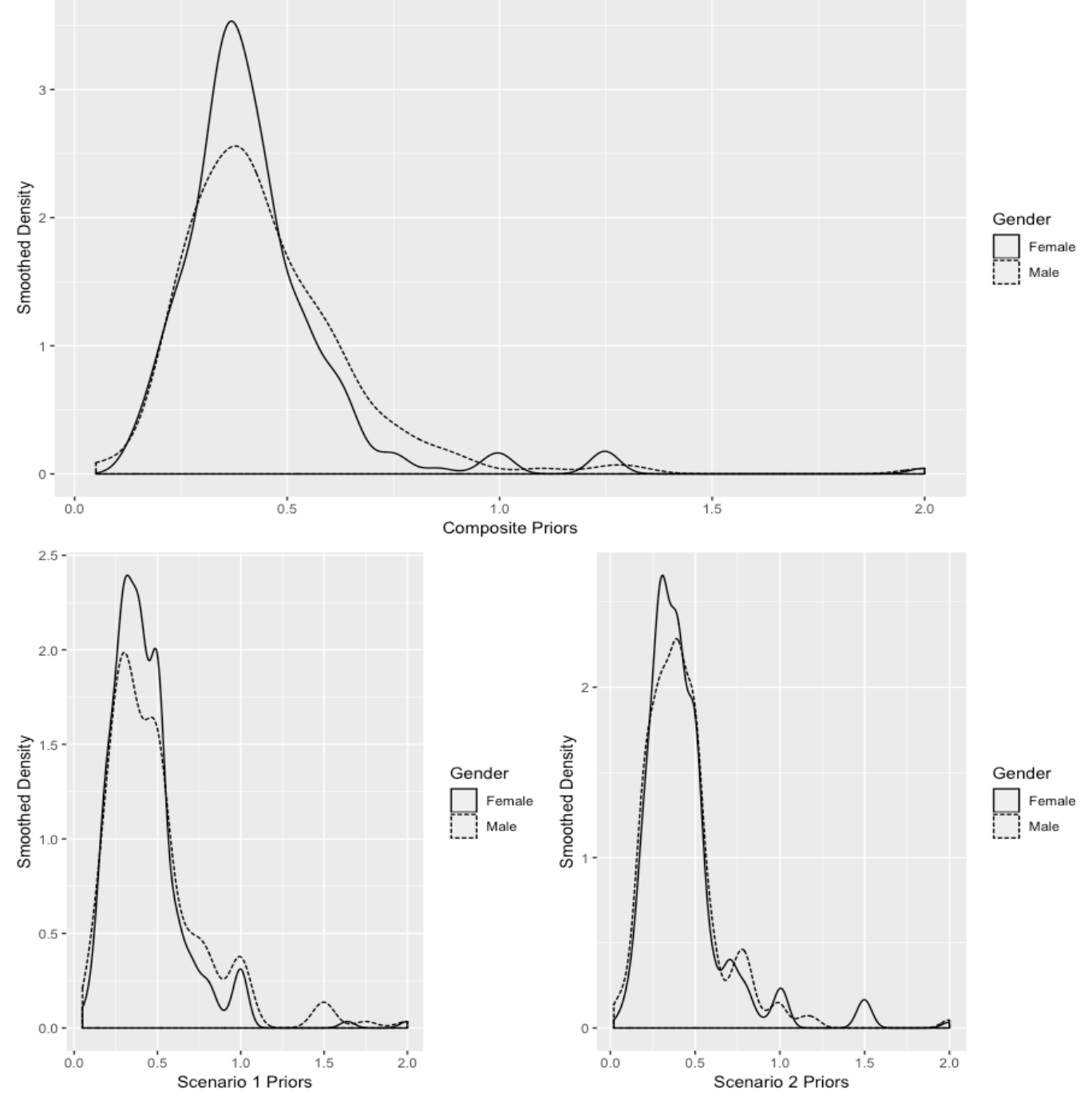

Figure 5. The density distributions of prior means chosen by women and men in Study 2 .

Note. The densities were smoothed using Gaussian kernel density estimation. 
PERSONAL CHARACTERISTICS AND STATISTICAL INFERENCES

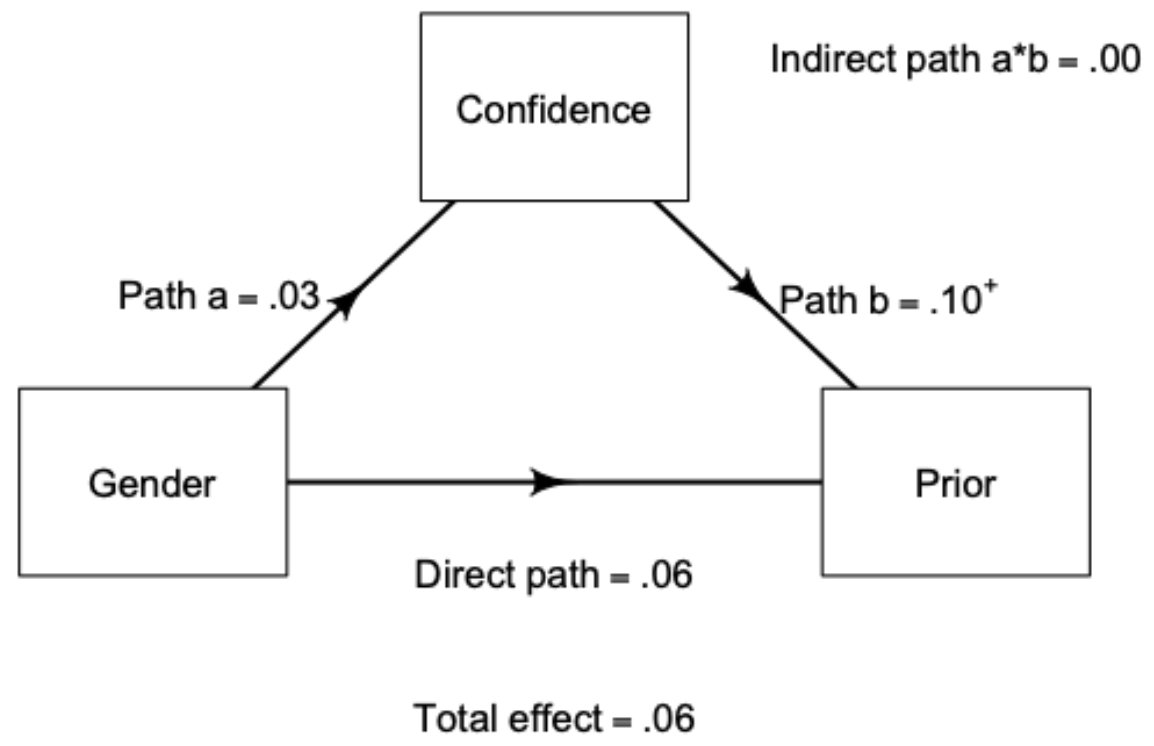

Figure 6. Path diagram of gender on the Real Research composite prior mean mediated by selfconfidence in Study 2.

Note. ${ }^{+}: p<.10$; otherwise: $p \geq .10$. 
PERSONAL CHARACTERISTICS AND STATISTICAL INFERENCES
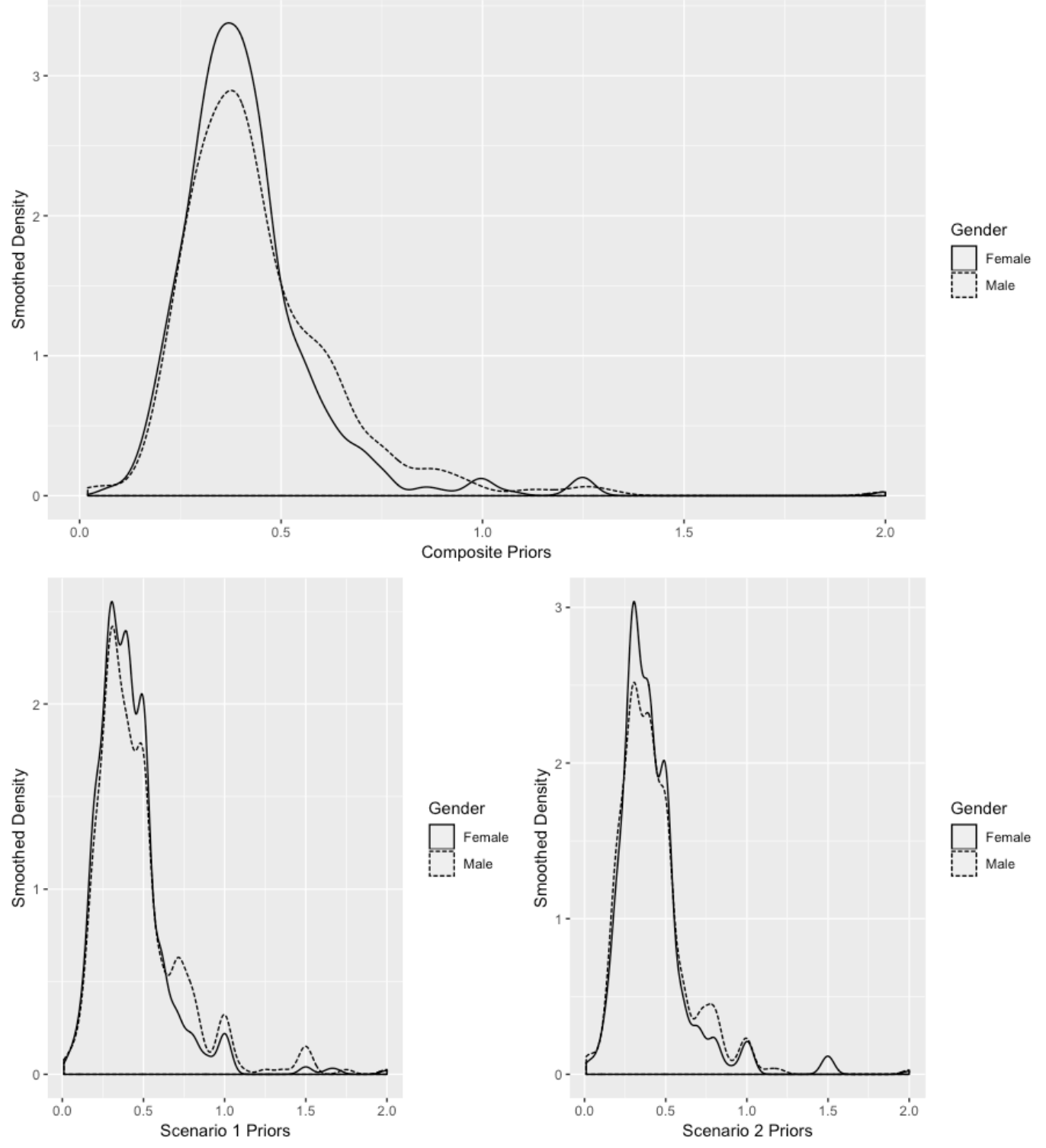

Figure 7. The density distributions of prior means chosen by women and men in the combined sample (Study $1 \& 2$ ).

Note. The densities were smoothed using Gaussian kernel density estimation. 
PERSONAL CHARACTERISTICS AND STATISTICAL INFERENCES

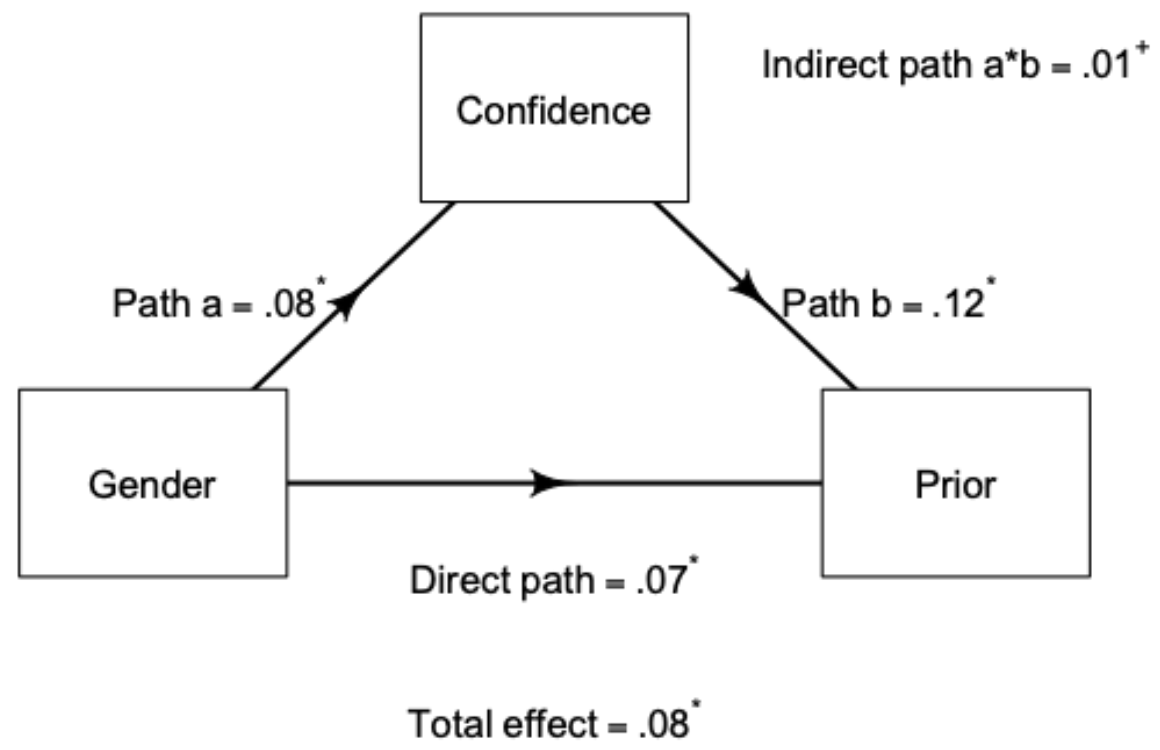

Figure 8. Path diagram of gender on the Real Research composite prior mean mediated by selfconfidence in the combined sample (Study $1 \& 2$ ).

Note. ${ }^{*} p<.05 ;{ }^{+}: p<.10 ;$ otherwise: $p \geq .10$. 
PERSONAL CHARACTERISTICS AND STATISTICAL INFERENCES

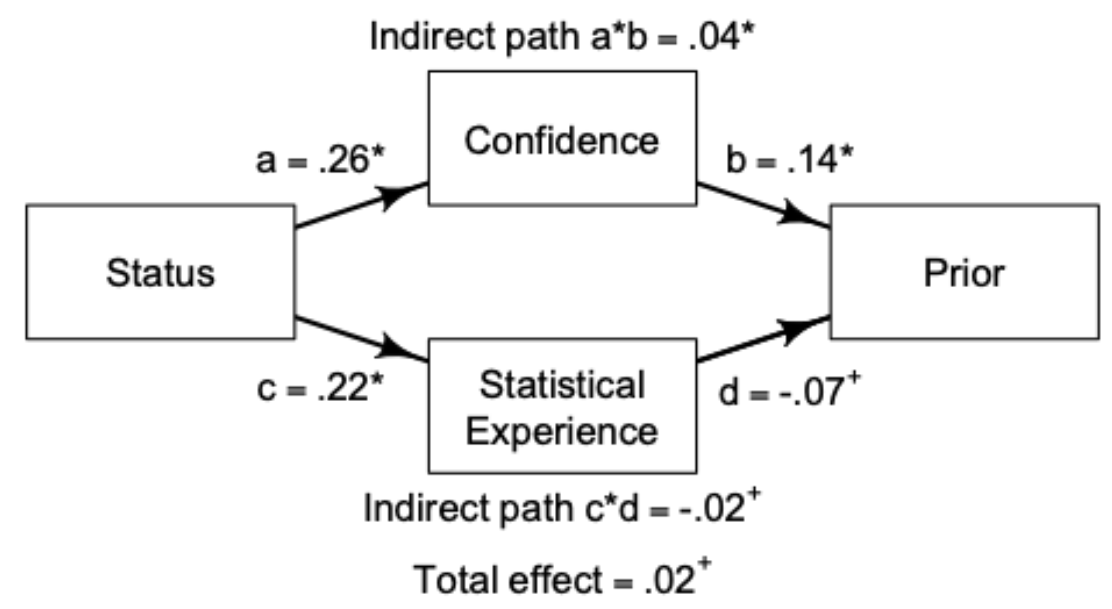

Figure 9. Path diagram of status on the Real Research composite prior mean mediated by researcher confidence and previous statistical experience in the combined sample (Study $1 \& 2$ ).

Note. ${ }^{*} p<.05 ;{ }^{+}: p<.10$; otherwise: $p \geq .10$. Confidence and statistical experience were allowed to correlate $(\beta=.19, p<.001)$. 